\title{
Overexpression of Polycomb-Group Gene rae28 in Cardiomyocytes Does Not Complement Abnormal Cardiac Morphogenesis in Mice Lacking rae28 But Causes Dilated Cardiomyopathy
}

\author{
Hideyuki Koga, Yoshikazu Kaji, Kiyomasa Nishii, Manabu Shirai,
} Daihachiro Tomotsune, Tomoaki Osugi, Akihisa Sawada, Ji Yoo Kim, Junichi Hara, Takeshi Miwa, Keiko Yamauchi-Takihara, Yosaburo Shibata, and Yoshihiro Takihara

Department of Developmental Biology and Medicine (HK, MS, TO, AS, JYK, YT), Osaka Medical Center for Cancer and Cardiovascular Diseases, Osaka, Department of Medical Genetics (HK, MS, DT, TO, AS, JYK, YT), Research Institute for Microbial Diseases, and Department of Genome Informatics (MS, TM), Genome Information Research Center, Osaka University, Suita, Departments of Molecular Medicine (TO, KY-T) and Developmental Medicine (AS, $J Y K, J H)$, Osaka University Graduate School of Medicine, Suita, and Departments of Medicine and Biosystemic Sciences (HK, YK) and Developmental Molecular Anatomy (KN, YS), Kyushu University Graduate School of Medical Science, Fukuoka, Japan

SUMMARY: The Polycomb-group genes (PcG) are widely conserved from Drosophila to mammals and are required for maintaining positional information during development. The rae28 gene (rae28) is a member of the mouse PcG. Mice deficient in rae28 $\left(\right.$ rae $\left.28^{-1-}\right)$ demonstrated that rae28 has a role not only in anteroposterior patterning but also in cardiac morphogenesis. In this study we generated transgenic mice with ubiquitous or cardiomyocyte-specific exogenous rae28 expression. Genetic complementation experiments with these transgenic mice showed that ubiquitous expression of rae28 could reverse the cardiac anomalies in rae28 ${ }^{-/}$, whereas cardiomyocyte-specific expression of rae28 could not, suggesting that rae28 is involved in cardiac morphogenesis through a noncardiomyocyte pathway. Interestingly, however, cardiomyocyte-specific overexpression of rae28 caused dilated cardiomyopathy, which was associated with cardiomyocyte apoptosis, abnormal myofibrils, and severe heart failure. Cardiac expression of rae28 was predominant in the early embryonic stage, whereas that of the other PcG members was relatively constitutive. Because rae28 forms multimeric complexes with other PcG proteins in the nucleus, it is presumed that constitutive cardiomyocyte-specific rae28 overexpression impaired authentic PcG functions in the heart. rae28-induced dilated cardiomyopathy may thus provide a clue for clarifying the direct role of PcG in the maintenance of cardiomyocytes. (Lab Invest 2002, 82:375-385).

$T$ he cardiac primordia originate in the lateral mesoderm, known as the cardiac crescent. The primitive heart tube appears as a single tube between 7.5 and 8 days post coitus (dpc) and undergoes looping and septation to complete cardiac morphogenesis. Although several families of transcription factors are now known to play critical roles in myocardial development and patterning (Srivastava and Olson, 2000), little is known yet about how these genetic programs are governed and the lifelong cardiac function is maintained. The rae28 gene (rae28) is a member of the mouse Polycomb-group

Received August 29, 2001.

This work was supported by a Grant-in-Aid for Scientific Research from the Ministry of Education, Science, Sports and Culture of Japan and grants from the Ministry of Health and Welfare of Japan.

Address reprint requests to: Dr. Yoshihiro Takihara, Department of Developmental Biology and Medicine, Osaka Medical Center for Cancer and Cardiovascular Diseases, 3-3, Nakamichi-1, Higashinari, Osaka 5378511,Japan.E-mail: takihara-yo@mc.pref.osaka.jp genes $(P c G)$. As previously reported, we generated mutant mice deficient in the rae28 gene $\left(\right.$ rae2 $\left.^{-1-}\right)$ that showed cardiac defects as well as posterior transformation of the skeletons and systemic neural crest defects caused by the altered expression of Hox genes during the maintenance phase in the paraxial mesoderm and rhombomeres (Takihara et al, 1997). Because rae28 ${ }^{-/-}$ displayed cardiac anomalies (including atrial septal defects, ventricular septal defects, tetralogy of Fallot, and double outlet of the right ventricle) that are representative congenital heart diseases in humans, rae28-deficient mice provided a first clue for identification of the role of PcG in heart development and function.

PcG are conserved between Drosophila and mammals. PcG proteins associate with each other through conserved domains and form multimeric complexes (Alkema et al, 1997; Hashimoto et al, 1998; Takihara and Hara, 2000; Tomotsune et al, 1999). There exist at least two classes of PcG complexes, PcG complexes 1 and 2 (Takihara and Hara, 2000). PcG complex 1 
consists of multimeric complexes, including PcG family members, M33, bmi1, mel18, and rae28 (also designated as mph1), mph2, and Scmh1 (Alkema et al, 1997; Hashimoto et al, 1998; Tomotsune et al, 1999) and may be a homolog to Drosophila Polycomb repressive complex 1 , which competitively antagonizes the ATP-dependent nucleosome remodeling ability of SWI/SNF family complexes (Shao et al, 1999). PcG complex 2 is distinct from PcG complex 1 and includes eed and Enx1/EZH2 or Enx2 (Takihara and Hara, 2000). Interestingly, PcG complex 2 interacts with histone deacetylase through eed and starts functioning at an earlier developmental stage than does PcG complex 1 (Schumacher et al, 1996).

As reported previously, mice deficient in bmi1 (van der Lugt et al, 1994), mel18 (Akasaka et al, 1996), M33 (Córe et al, 1997; Katoh-Fukui et al, 1998), rae28 (Takihara and Hara, 2000), and ring1A (del Mar Lorente et al, 2000) revealed transformations along the anteroposterior (A-P) axis, similar to PcG mutations in flies, which are presumed to be caused by the altered expression of Hox genes. In addition, mutations in eed were also found to lead to impaired A-P patterning at an earlier stage (Schumacher et al, 1996). Thus, PcG in flies and mice have similar functions in that they have a role in maintaining positional information. Recently, accumulating evidence suggests that mammalian $P C G$ genes are involved in a greater variants of functions than are Drosophila PCG genes (Takihara and Hara, 2000).

For this study we generated transgenic mice overexpressing rae28 specifically in cardiomyocytes as well as those expressing rae28 ubiquitously to address the question to what extent and how rae28 is involved in cardiac development and functiōn. We ussed a moūse $\beta$-myosin heavy chain $(\beta M H C)$ promoter (Rindt et al, 1993) and a chicken $\beta$-actin promoter with a Rous sarcoma virus (RSV) enhancer (Wakamatsu et al, 1997) to drive exogenous rae28 expression in cardiomyocytespecific and ubiquitous manners, respectively. Ubiquitous rae28 expression reversed abnormalities in rae $28^{-1-}$ cardiac phenotype, whereas cardiomyocytespecific rae28 overexpression could not. Interestingly, however, cardiomyocyte-specific rae28 overexpression impaired cardiomyocytes, causing dilated cardiomyopathy (DCM). Thus we argue for the importance of the $P C G$ genes in the lifelong maintenance of cardiomyocytes as well as in a noncardiomyocyte pathway regulating cardiac morphogenesis.

\section{Results}

\section{Generation of Transgenic Mice Expressing Exogenous rae28}

Because neonatal rae28 $8^{-/}$mice displayed cardiac anomalies compatible with human congenital heart diseases, we attempted to analyze how rae28 is involved in cardiac morphogenesis during development. We generated transgenic mice carrying a fulllength Rae28 cDNA, whose expression was driven either by a chicken $\beta$-actin promoter with an RSV enhancer (Wakamatsu et al, 1997) or by a mouse $\beta M H C$ promoter (Rindt et al, 1993). These transgenic mice were designated as RSV-rae28 and $\beta M H C$ rae28, respectively. Two independent founder lines of $\beta M H C$-rae28 and three lines of RSV-rae28 were generated. The numbers of the transgenes were estimated to be 2 and 6 in $\beta M H C$-rae28 and 4, 4, and 6 in $R S V$-rae28 by Southern blot analysis (data not shown). These transgenic mice were genetically backcrossed to C57BL/6 strain mice to minimize the effects of their genetic background. To examine exogenous rae28 expression derived from these transgenes, these transgenic mice were genetically intercrossed with rae $28^{+/-}$, and the resulting offspring were backcrossed to rae28 $8^{+/-}$to obtain rae28 ${ }^{-/-}$carrying either the $R S V$-rae28 or $\beta M H C$-rae28 transgene, because expression of rae28 was barely detectable in rae28 ${ }^{-1-}$ embryos. We examined rae28 expression in these mice by means of whole-mount in situ hybridization and confirmed that exogenous rae28 expression reflected the specificity of each of the promoters and enhancers (Fig. 1A). RSV-rae28/rae28 $8^{-1-}$ embryos showed ubiquitous expression of exogenous rae28 (Fig. 1A, lower left panel), whereas BMHC-rae28/ rae28 $8^{-1-}$ embryos showed heart-specific expression of rae28 associated with weaker rae28 expression in the somites (Fig. 1A, lower right panel). Cardiomyocyte-specific rae28 expression was confirmed by section in situ hybridization of the heart derived from $\beta M H C-r a e 28 / r^{2} 28^{-/-}$(not shown). Northern blot analysis also detected exogenous expression of rae28 in the heart and weak expression in the skeletal muscle of the adult $\beta M H C$-rae28 (Fig. 1B). The expression level of rae28 in RSV-rae28/rae28 ${ }^{-1-}$ whole embryos was weaker than that in the wild type, and cardiac expression of rae28 was much weaker in $R S V$-rae28 than in $\beta M H C$-rae28 (Fig. 1A). We confirmed expression of rae28 protein in these transgenic mice by Western blot analysis. In 17.5-dpc $\beta M H C$ rae28 hearts, two bands were detected that were identical to those in 13.5-dpc whole embryos (Fig. 1C), whereas weaker rae28 expression was detected in RSV-rae28 hearts (not shown). Exogenous rae28 expression was detected even in 6-month-old $\beta M H C$ rae28 hearts but was weaker than that in 17.5-dpc ones (Fig. 1C). We confirmed for each of these transgenic founders that the transgenic animals derived from them had essentially similar expression profiles and phenotypes.

\section{Histoanatomical Examination of rae28 ${ }^{-1-}$ Complemented by Exogenous rae28 Expression}

To examine whether exogenous expression of rae28 affected rae28 $8^{-1-}$ phenotype, we analyzed $\beta M H C$ -

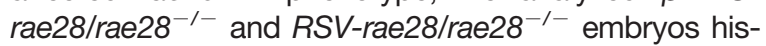
tologically. We observed 32 RSV-rae28/rae28 ${ }^{-/-}$neonates, all of which were as viable as wild type until the adult stage and were fertile. We found no abnormal morphologic findings in the hearts from 32 of the animals (Table 1). The other phenotypes, including systemic neural crest defects, skeletal posterior transformation, 

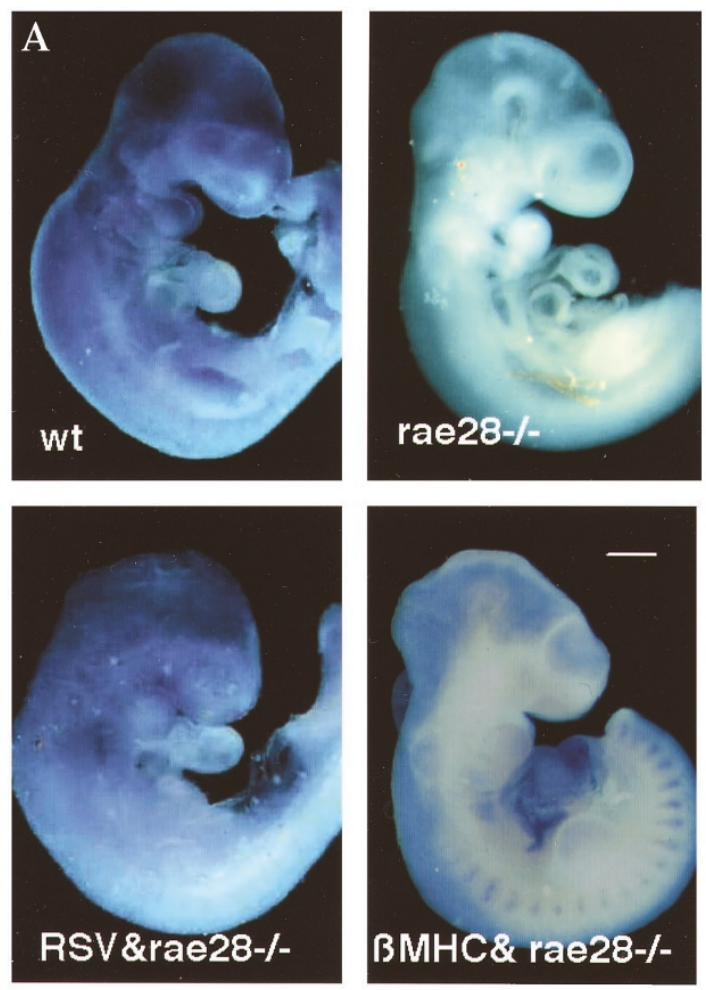

B

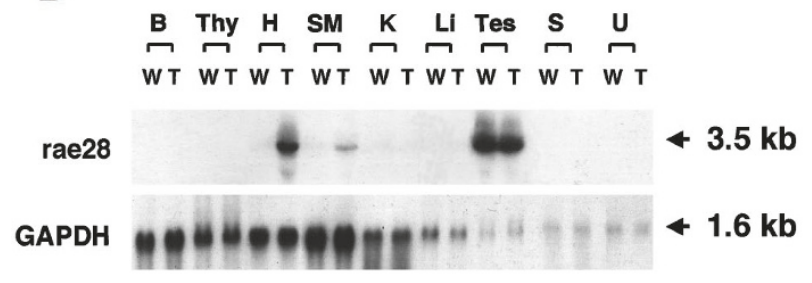

C

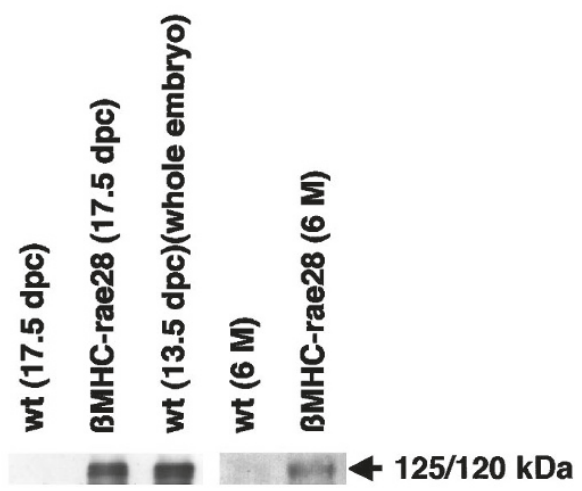

Figure 1.

Expression of exogenous rae28 in Rous sarcoma virus (RSV)-rae28 and $\beta$-myosin heavy chain ( $\beta M H C)$-rae28. A, Expression of rae28 was detected in 9.5-dpc embryos by means of whole-mount in situ hybridization analysis. Wt $=$ Wild type; rae28- ${ }^{-\prime}=$ Homozygous rae28-deficient embryo; RSV\&rae28-I-,

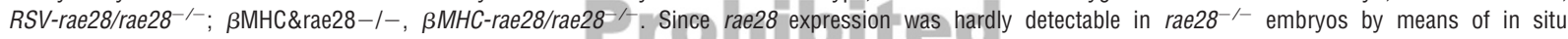
hybridization, detected rae28 expression can be assured to correspond to exogenous rae28 expression derived from the transgenes. Scale bar, $500 \mu \mathrm{m}$. B, Northern blot analysis of 6-month-old $\beta M H C$-rae28. Total cellular RNAs were extracted from each of the organs and subjected to Northern blot analysis. GAPDH expression is shown to determine the amount of RNAs on the filter. $\mathrm{W}=$ Wild type; $\mathrm{T}=\beta M H C$-rae28; $\mathrm{B}=\mathrm{Brain}$; Thy = Thymus; $\mathrm{H}=\mathrm{Heart} ; \mathrm{SM}=\mathrm{Skeletal}$ muscle; $\mathrm{K}=$ Kidney; $\mathrm{Li}=$ Liver; Tes = Testis; $\mathrm{S}=$ Spleen; $\mathrm{U}=$ Uterus. The sizes of the detected bands are shown on the right side of the panels. Heart-specific expression derived from the $\beta M H C$-rae28 transgene was clearly detected, whereas weak expression was also detectable in the skeletal muscle. Note that endogenous rae28 expression was undetectable in adult tissues except for the testis. C, Western blot analysis of the heart. The hearts were removed from 17.5-dpc embryos and from 6-month-old mice and subjected to Western blot analysis with a polyclonal antibody against rae28 protein. Two bands with molecular weight of 125 and $120 \mathrm{kDa}$ were detected in $\beta M H C$-rae28 hearts. These bands were compatible with those in 13.5-dpc whole embryos as shown in the figure. We detected rae28 in the wild-type heart by overexposure (not shown). $\mathrm{Wt}=$ Wild type; $\mathrm{M}=$ Month.

and splenic hypoplasia, also disappeared (Table 1), indicating that this full-length rae28 cDNA encodes the functional rae 28 protein and that ubiquitous expression of rae28 complemented the lack of endogenous rae28 expression. However, histologic examination of the hearts from $12 \beta M H C-r a e 28 / r^{2} 28^{-/-}$embryos revealed that all of them showed cardiac anomalies similar to those observed in rae28 $8^{-1-}$, which included membranous or muscular ventricular septal defects, atrial septal defects, and tetralogy of Fallot (Table 1). Cardiomyocyte- specific rae28 expression thus showed no effect on abnormal cardiac morphogenesis in rae $28^{-/-}$, whereas ubiquitous rae28 expression could reverse the rae28 $8^{-/-}$ phenotype, suggesting that rae28 is involved in cardiac morphogenesis through a noncardiomyocyte pathway.

\section{Prognosis and Histologic Examinations of $\beta \mathrm{MHC}-\mathrm{rae28}$}

$R S V-r a e 28$ showed no histologic abnormalities in the heart and had a lifespan compatible with that of 
Table 1. Effect of Exogenous Expression of rae28 on Cardiac Anomalies in rae28 ${ }^{-/-}$

\begin{tabular}{cc}
\hline Lines & $\begin{array}{c}\text { Animals with } \\
\text { reversion/examined }\end{array}$ \\
\hline RSV-rae28/rae28 & $32 / 32$ \\
$\beta M H C-r a e 28 /$ rae28 $^{-/-}$ & $0 / 12$ \\
\hline
\end{tabular}

rae28 $8^{-1}$ embryos with each of the transgenes were examined histoanatomically, and the numbers of animals with reversion of cardiac phenotypes are shown. In addition to reversion of the cardiac anomalies, all the other phenotypes, posterior transformation of the skeletons, systemic neural crest defects, and splenic hypoplasia were also reversed in $R S V$-rae28/rae28 ${ }^{-1-}$.

wild-type animals (Fig. 2), whereas $\beta M H C$-rae28 died in the early adult stage. We observed $35 \beta M H C$-rae28. All of the $35 \beta M H C$-rae28 we observed were lethal within 8 months, as shown in the Kaplan-Meier survival curve (Fig. 2), whereas littermates with a wildtype genotype showed no difference in lifespan from C57BL/6 strain mice. $\beta M H C$-rae28 showed typical findings compatible with heart failure, ie, general weakness, lack of food and water intake, labored breathing, and generalized edema with marked gain of body weight. Death was, thus, preceded for several days by generalized edema and dyspnea as described above.

To evaluate cardiac function in vivo, 5- to 6-monthold $\beta M H C-r a e 28$ were subjected to transthoracic M-mode echocardiography. The results showed that the left ventricular (LV) dimension was significantly increased, and the LV ejection fraction was also significantly decreased in $\beta M H C-r a e 28$ as compared with the wild type (Table 2 and Fig. 3). No reduction in LV posterior wall thickness and interventricular septal thickness was detectable at this stage.

Although we observed no abnormal anatomical findings in the hearts from neonatal $\beta M H C-r a e 28$, at autopsy the hearts were markedly enlarged with severe reduction of the ventricular wall thickness (Fig. $4 \mathrm{~A}$, lower panel), and intracavitary thrombi were frequently present in the left atrium (not shown). Hypertrophy of the heart was not observed at any developmental stage of the $\beta M H C$-rae28. These animals also revealed a marked pleural effusion (Fig. 4A, upper

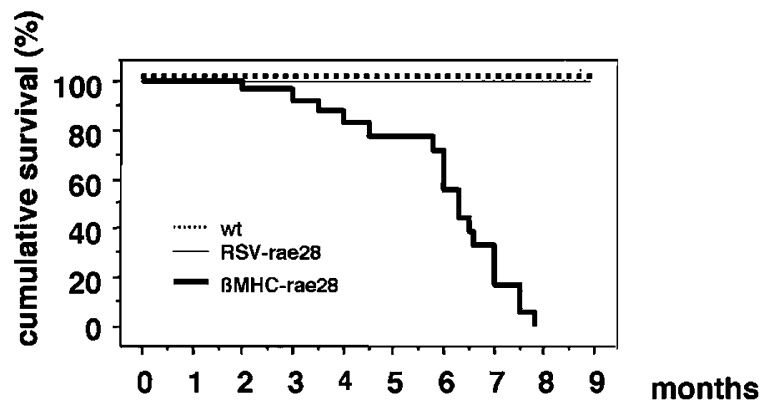

Figure 2.

Survival of $\beta M H C$-rae28 and RSV-rae28. Mortality rates from a total of 35 $\beta M H C$-rae28, 33 RSV-rae28, and 30 littermates with a nontransgenic genotype were estimated by Kaplan-Meier survival analysis. The survival curve shows a significantly shorter lifespan for $\beta M H C$-rae28 $(p<0.05)$. Wild type, dotted line; $\beta M H C$-rae28, bold solid line; RSV-rae28, thin solid line.
Table 2. Echocardiographic Indices

\begin{tabular}{lcc}
\hline \multicolumn{1}{c}{ rae28 } & Wild type & $\beta M H C-$ \\
\hline & $(\mathrm{n}=8)$ & $(\mathrm{n}=5)$ \\
Heart rate (beat per min) & $319 \pm 89$ & $300 \pm 53$ \\
LV end-diastolic & $4.1 \pm 0.5$ & $5.1 \pm 0.6^{*}$ \\
$\quad$ dimension (mm) & & \\
LV end-systolic & $2.7 \pm 0.4$ & $3.7 \pm 0.5^{* *}$ \\
$\quad$ dimension (mm) & & \\
IVS thickness (mm) & $0.7 \pm 0.1$ & $0.8 \pm 0.1$ \\
LV posterior wall & $0.7 \pm 0.1$ & $0.7 \pm 0.1$ \\
$\quad$ thickness (mm) & & \\
Ejection fraction (\%) & $62 \pm 10$ & $52 \pm 5^{*}$ \\
\hline
\end{tabular}

Five- to 6-month-old $\beta M H C$-rae28 and wild type mice were subjected to M-mode echocardiography. Wild type versus $\beta M H C$-rae28 were compared using the unpaired Student's $t$ test. ${ }^{\star} p<0.05,{ }^{\star \star} p<0.01$ vs. wild type. BW, body weight; LV, left ventricular; IVS, interventricular septal.

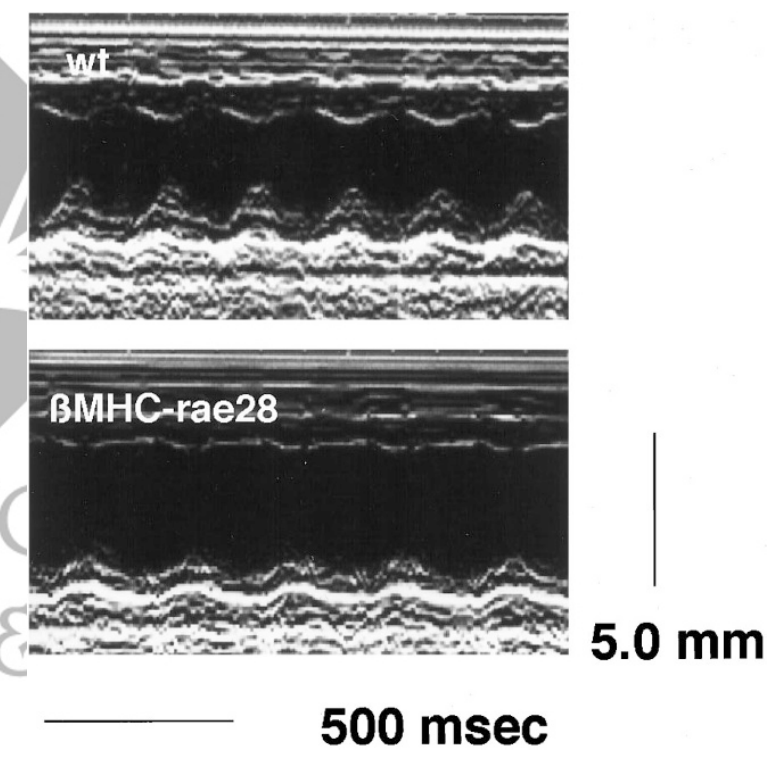

Figure 3.

Transthoracic M-mode echocardiography. Representative tracings from 5-month-old $\beta M H C$-rae28. Wt $=$ wild type; $\beta M H C$-rae28 $=$ a $\beta M H C$-rae28 transgenic mouse. See Table 2 for a compilation of the measured indices.

panel) and ascites (not shown), indicating that $\beta M H C$ rae28 featured decompensated states of congestive heart failure. Examination of $\beta M H C$-rae28 kidneys showed no abnormal findings (not shown). In pursuit of evidence of abnormalities in cell ultrastructure, the hearts of 3- and 6-month-old $\beta M H C$-rae28 were subjected to electron microscopic analysis. We chose apparently healthy mice to evaluate primary effects of the transgene on the cardiomyocytes (Fig. 4B). We found no significant difference in the atria. By contrast, most of the $\beta M H C$-rae28 ventricles were occupied by abnormal cardiomyocytes in both of the 3- and 6 -month cases. Their sarcomeres showed abnormal arrangements with irregular and loose myofilaments; $Z$ lines were vague and enlarged. In the severest regions, $\mathrm{H}$ and $\mathrm{M}$ lines were not visible. In addition to the abnormalities of the myofilaments, the number of the stacks of the mitochondrial cristae was reduced in 

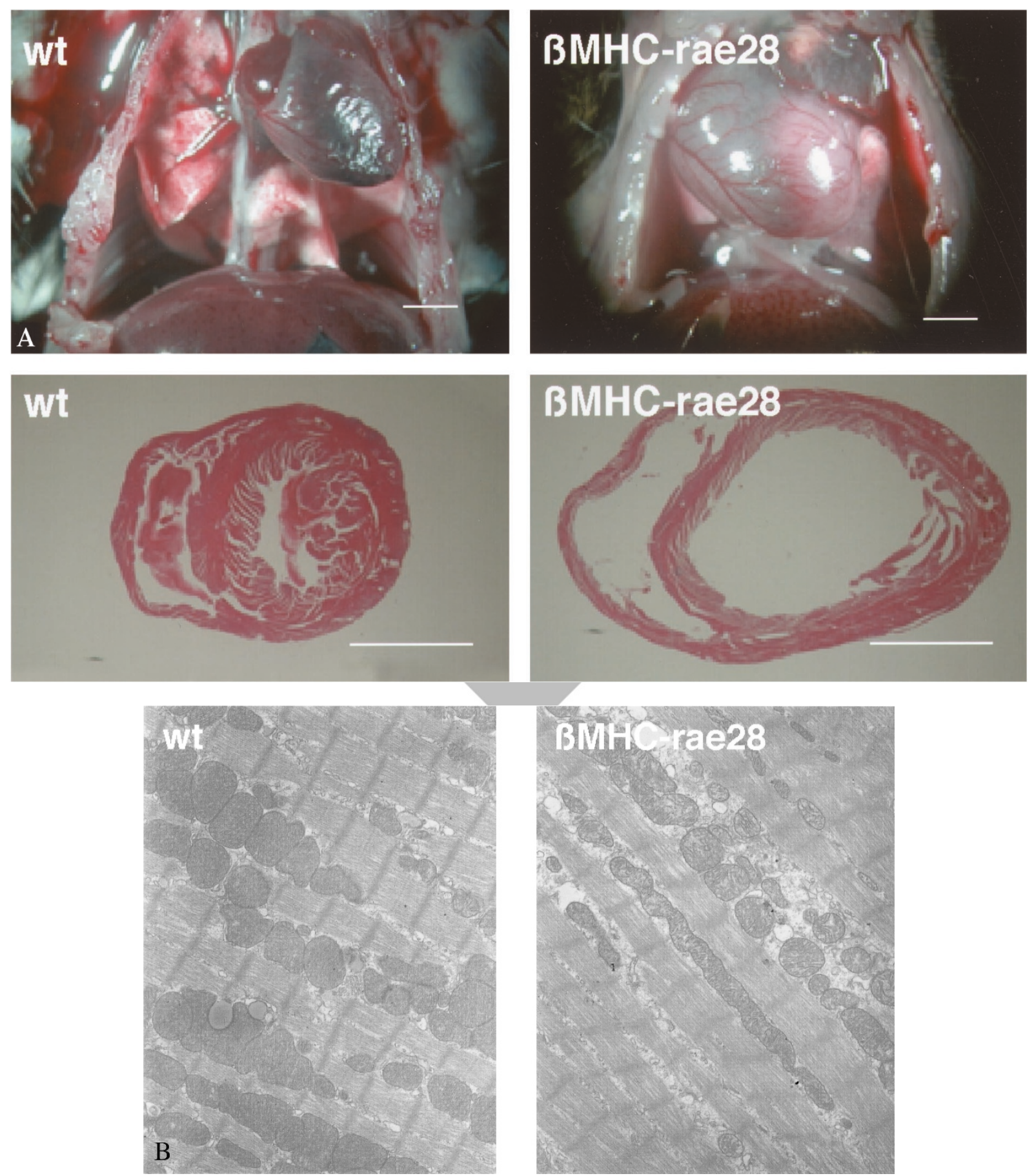

Figure 4.

Histoanatomical findings of $\beta M H C$-rae28 with heart failure. A, External appearances of the hearts are shown in the upper panels. The anterior thoracic wall was removed to allow the interior of thoracic chamber to be observed. Transverse sections with hematoxylin-eosin stain are shown in the lower panels. The hearts were removed from wild-type and 7-month-old $\beta M H C$-rae28 and were examined histologically. Note marked dilation and reduced wall thickness in both of the ventricles and massive pleural effusion in $\beta M H C$-rae28. Wt $=$ Wild type. Scale bar, $2 \mathrm{~mm}$. B. Electron microscopic analysis of the ventricular myocardium of 3-month-old wild type and $\beta M H C$-rae28. In contrast to the regular arrays of the wild-type sarcomeres, irregularity of the mutant myofibrils are prominent. Shown in $\beta M H C$-rae28 is a so-called giant mitochondrion (Ghadially, 1988), which was not found in the wild-type hearts. We observed similar findings in 6-month-old $\beta M H C$-rae28 (not shown).

$\beta M H C$-rae28. We sometimes found very elongated mitochondria, which were not detected in the wildtype hearts. These mitochondrial abnormalities are caused by various biomechanical stresses, such as hypoxia and myopathies (Ghadially, 1988). Taken together, these ultrastructural findings correlate well with the features of human DCM (Tashiro et al, 1990). Van Gieson staining did not detect any cellular infiltration or fibrous changes (not shown). We further examined apoptosis in $\beta M H C$-rae28 hearts by TUNEL (terminal deoxynucleotidyl transferase-mediated dUTP nick-end labeling) staining while the nucleus was 

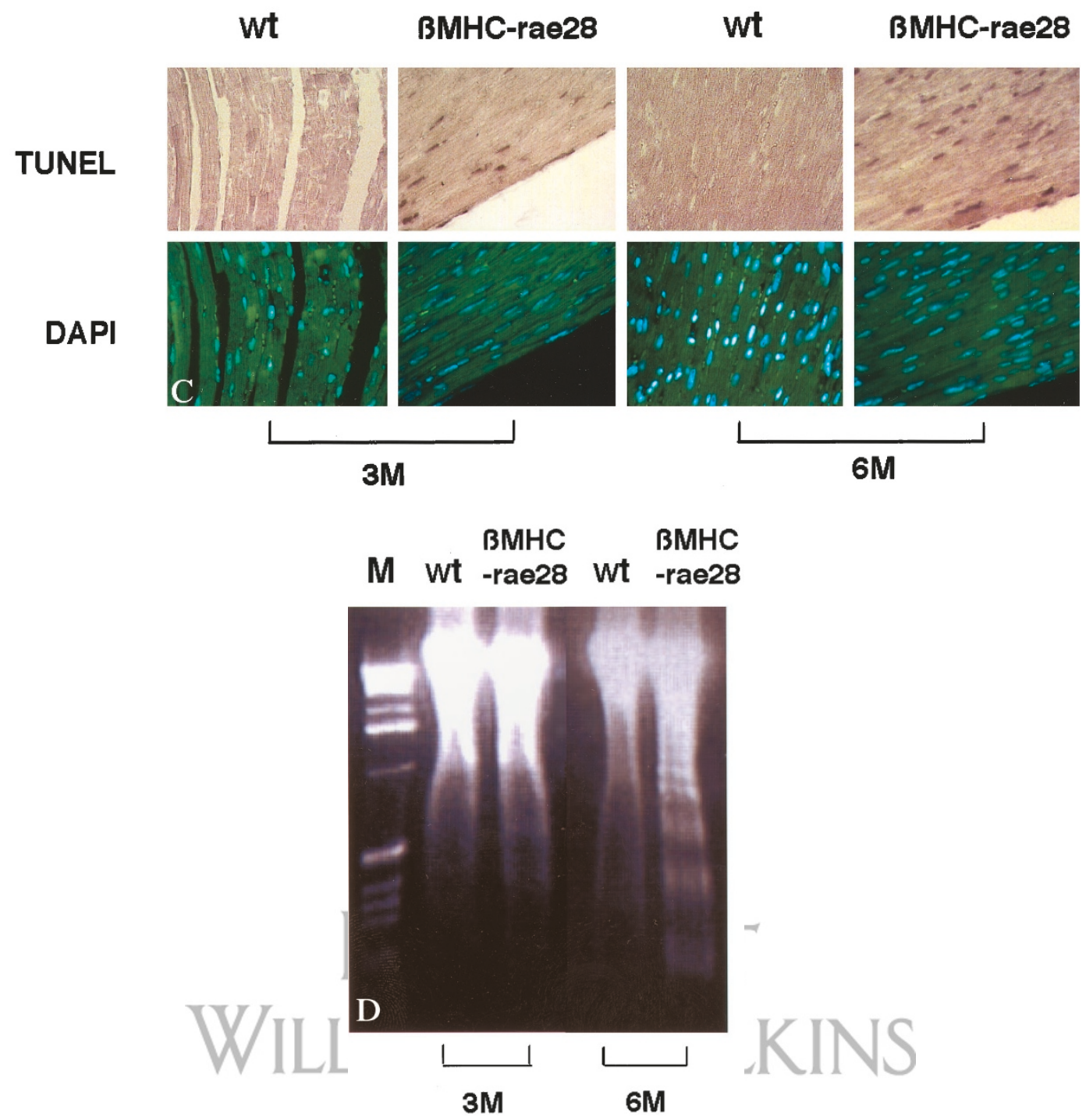

Figure 4.

C, TUNEL staining of the hearts from the 3 - and 6-month-old wild type and $\beta M H C$-rae28. The sections from these hearts were stained with the TUNEL method (upper panels) and with 4,6-diamidino-2-phenylindole (lower panels). TUNEL-positive cardiomyocytes were detected in 3-month-old $\beta M H C$-rae28 and had remarkably increased in the 6 -month-old animals. D, DNA ladder analysis. High molecular weight DNAs were isolated and analyzed by $1 \%$ agarose electrophoresis. $\mathrm{M}=\mathrm{Molecular}$ marker; wt $=$ Wild-type heart; $\beta M H C$-rae28 $=\beta M H C$-rae28 heart.

\section{Problolited}

simultaneously stained with 4,6-diamidino-2-phenylindole (Fig. 4C). We detected TUNEL-positive cardiomyocytes in 3-month-old $\beta M H C$-rae28 hearts as well as in 6 -month-old ones. About $8 \%$ of these cardiomyocytes were already TUNEL positive in 3-month-old $\beta M H C$-rae28 hearts. Similar findings were observed in both the right and left ventricles. TUNEL-positive cardiomyocytes had markedly increased to $25 \%$ in 6-month-old $\beta M H C$-rae28 hearts, when heart failure was in progress and caused increasing mortality. We also confirmed the presence of apoptotic cardiomyocytes by DNA ladder analysis (Fig. 4D). Fragmented DNA was detectable in 3-month-old $\beta M H C$-rae28 hearts and had increased in the 6-month-old ones, which was consistent with the findings for the TUNEL staining (Fig. 4, C and D). Examination of isolated cardiomyocytes from neonatal $\beta M H C$-rae28 by
Annexin-V staining did not detect any significant apoptotic cells at these stages (not shown). It was thus presumed that apoptosis started in $\beta M H C$-rae28 hearts in the pup stage before heart failure occurred. Although many $\beta M H C$-rae28 cardiomyocytes were TUNEL positive, cells with typical apoptotic morphology were very rare in the electron microscopic analysis on the $\beta M H C$-rae28 cardiomyocytes (not shown), probably because the process of myocyte apoptosis runs its course in a short time.

\section{Expression of Molecular Markers Induced in BMHC-rae28 Hearts}

We examined the cardiac expression of molecular markers that are known to be affected by biomechanical stress such as heart failure. Total RNAs from the 
hearts from 6-month-old animals were extracted and were subjected to semiquantitative RT-PCR analysis. Significant up-regulation of $\beta M H C$, atrial natriuretic factor (ANF), and skeletal $\alpha$-actin transcripts was observed in $\beta M H C$-rae28 hearts compared with those in wild-type ones, whereas cardiac $\alpha$-actin and $\alpha-M H C$ transcripts were down-regulated (Fig. 5). These findings indicate that biomechanical stress was induced, and cellular responses to the stress were elicited in $\beta M H C$-rae28 hearts.

\section{Expression of PcG Members in the Heart During Mouse Development}

Total cellular RNAs were extracted from the hearts of 8.5-, 14.5-, and 18.5-dpc embryos and 3-month-old mice with C57BL/6 genetic background. These RNAs were subjected to RT-PCR analysis with oligonucleotide primers as described under "Materials and Methods." Expression of rae28 was high in 8.5-dpc embryonic hearts, whereas it decreased in the later embryonic stages. Very weak rae28 expression was, however, still detectable in the adult stage. On the other hand, expression of the other PcG genes, M33, mel18, bmi1, and Scmh1, remained relatively constitutive (Fig. 6A). No obvious change was detected in the expression of M33 and mph2. bmi1 and scmh1 expression seemed to slightly increase after $8.5 \mathrm{dpc}$, whereas mel18 expression decreased 3 months after birth. These findings indicated that PcG members other than rae28 were expressed in the heart not only during the embryonic stage but also in the adult. Interestingly, rae28 expression was strong in the early embryonic stage but markedly decreased in the later stages, suggesting the possibility that PcG complexes in the heart change in the constituents during heart development.

\section{Discussion}

We generated two kinds of transgenic lines that either expressed exogenous rae28 ubiquitously (RSV-rae28)

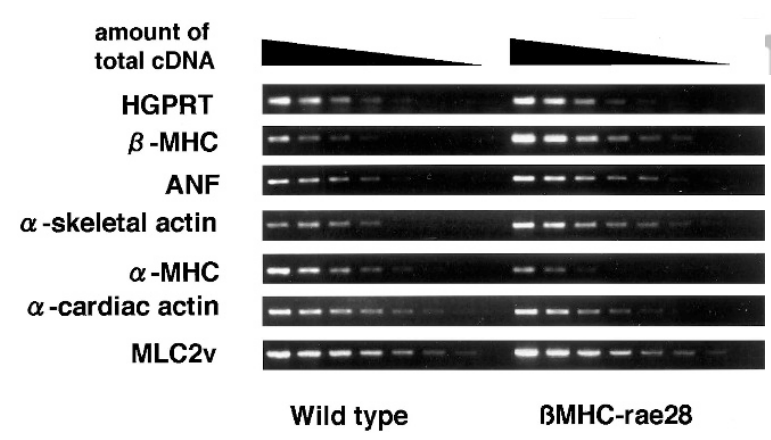

Figure 5.

Quantitative RT-PCR analysis of gene expression in wild-type and $\beta M H C$-rae28 hearts. As indicated by the wedges above the panel, 2-fold serial dilutions of cDNAs were used as templates for PCR amplification with primers specific for HGPRT, $\beta M H C$, ANF, skeletal and cardiac $\alpha$-actins, $\alpha$-MHC, and MLC2v. Signals for HGPRT mRNA were used as controls to adjust the amount of CDNAs. $\beta M H C, A N F$, and skeletal $\alpha$-actin transcripts were up-regulated in $\beta M H C$-rae28 heart, whereas cardiac $\alpha$-actin and $\alpha-M H C$ transcripts were down-regulated. The findings were confirmed by experiments in triplicate.
A

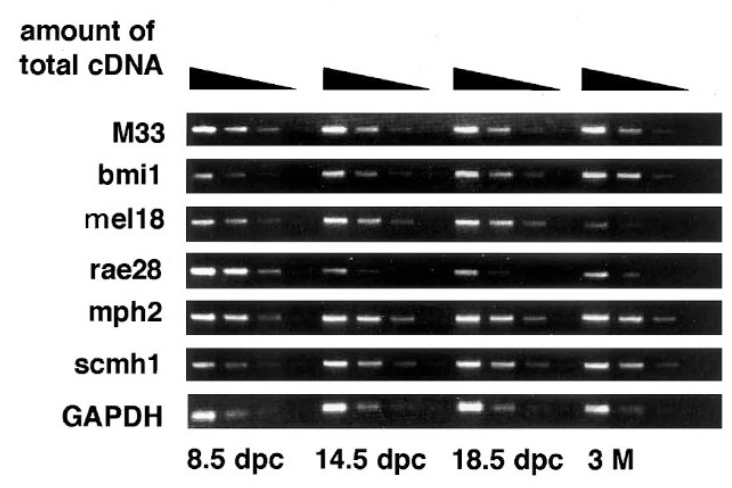

B

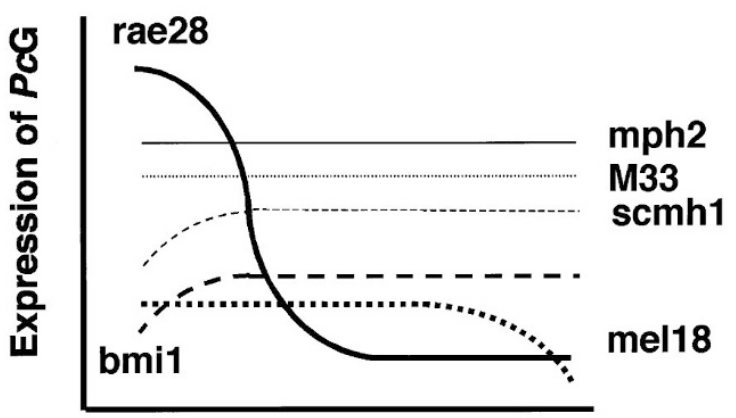

\section{5 dpc Birth $3 \mathrm{M}$}

Figure 6.

A, Expression of PCG genes in the heart during development. Total cellular RNAs were extracted from the heart and quantitative RT-PCR analysis was performed by using the oligonucleotide primers described in "Materials and Methods." Fourfold serial dilutions of cDNAs were used as templates for PCR amplification. Note that rae28 expression was high in the early embryonic stage but markedly decreased in the later stages. These findings were confirmed by experiments in triplicate. B, Expression profile of $P C G$ in the heart. The RT-PCR data shown in (A) were used for this schematic representation of the oscillations of $P C G$ expression.

or expressed it specifically in the cardiomyocytes ( $\beta M H C-r a e 28)$. Although endogenous $\beta M H C$ expression was restricted to the embryonic stage, we detected exogenous rae28 expression even in adult $\beta M H C-r a e 28$ hearts. Similar transcription activity of the $\beta M H C$ promoter has been reported previously (Rindt et al, 1993). However, it is presumed that expression of exogenous rae28 through the $\beta M H C$ promoter was also induced by biomechanical stress in $\beta M H C-r a e 28$, because endogenous $\beta M H C$ was induced significantly in 6-month-old $\beta M H C$-rae28 heart (Fig. 5).

By genetic intercrossing experiments, we showed that ubiquitous expression of rae28 completely complemented abnormal morphogenesis in rae28 ${ }^{-1-}$ heart but that the $\beta M H C$ promoter-driven cardiomyocyte-specific expression of rae28 could not rescue the cardiac phenotype even partially (Table 1). It is thus unlikely that $\beta M H C$-rae28 failed to produce a functional rae28 protein in the heart (Fig. 1C). Furthermore, cardiomyocytespecific rae28 overexpression did not seem to impair cardiac morphogenesis because $\beta M H C$-rae28 with the 
wild-type genetic background $\left(\right.$ rae $\left.28^{+/+}\right)$showed no abnormal cardiac morphology at least until severe heart failure occurred. Thus, rae28 plays an important role in cardiac morphogenesis via a noncardiomyocyte pathway. Because A-P patterning and neural crest development were affected in rae28 ${ }^{-1-}$ (Takihara et al, 1997), signals related to A-P patterning and/or those from cardiac neural crest cells might be a candidate for mediating the pathway. Recently we found that reduced $N k x 2.5 / C s x$ expression in the maintenance phase is responsible for cardiac anomalies in rae28 ${ }^{-1-}$ (Osugi et al, 2000). Investigations using these mutant animals are now in progress to determine how rae28 regulates Nkx2.5/Csx expression in the cardiomyocytes.

In this study we also provide evidence suggesting that PcG have a crucial role in the maintenance of cardiomyocytes. rae28 is strongly expressed in the heart at the early stages of development but is reduced drastically after $9.5 \mathrm{dpc}$ (Fig. 6B). Constitutive cardiomyocytespecific overexpression of rae28 resulted in DCM. $\beta M H C$-rae28 heart showed no gross morphologic abnormalities in the neonatal period but developed severe heart failure causing 100\% lethality within 8 months after birth (Fig. 2). DCM is a common form of heart failure in humans, whose molecular mechanisms underlying cardiomyocyte damage resulting in lethal cardiac failure are still poorly understood. The mice generated by us thus constitute a novel animal model for DCM, which is characterized by a development remarkably similar to the clinical course observed in patients with DCM. $\beta M H C-r a e 28$ heart revealed progressive cardiac failure associated with severe cardiac dilation and thinning of the myocardium (Fig. 4A). The cardiac structural and functional changes in $\beta M H C$-rae28 were also detected by echocardiography (Fig. 3). Thus, apoptosis and the affected myofibrils may act to account for the pathogenesis of cardiomyopathy in $\beta M H C$-rae28. Histoanatomical and physiologic examination of RSV-rae28 heart revealed no obvious differences observed between wild type and RSV-rae28 (not shown), and survival of RSVrae28 was similar to that of wild type (Fig. 2). Expression of exogenous rae28 was much weaker in $R S V$-rae28 hearts than in $\beta M H C$-rae28 hearts, which was also confirmed by RT-PCR analysis (not shown). The difference in exogenous rae28 expression levels in the heart may be the reason why DCM occurred in $\beta M H C$-rae28 but not in RSV-rae28. Concurrently with induction of endogenous $\beta M H C$ by the biomechanical stress, rae28 expression derived from the $\beta M H C-r a e 28$ transgene was also presumed to be induced, which could elicit further progress of DCM in $\beta M H C$-rae28. Although endogenous rae28 expression decreased earlier than 14.5 dpc, followed by constitutive exogenous rae28 expression in $\beta M H C-r a e 28$, it occurred later than 3 months when apoptosis and evidence for DCM were detected in the mice. This shows good consistency with the clinical course of DCM patients with inherited mutations in sarcomeric proteins.

It is generally presumed that cardiomyocyte hypertrophic and survival signals have a role in maintaining a compensatory phase of cardiomyocytes during biomechanical stress. So far, however, we do not have enough evidence to suggest molecular mechanisms that can account for cardiomyocyte-specific overexpression of rae28 resulting in apoptosis and the proceeding of DCM. Interestingly, however, expression of rae28 is down-regulated early in cardiac development, and only weak expression persists later in development and even in the adult stage, whereas the other PcG genes, M33, bmi1, mel18, mph2, and scmh1, showed relatively persistent expression in the heart (Fig. 6, A and B). As we reported previously, these PcG gene products form a multimeric complex designated as PcG complex 1 (Takihara and Hara, 2000) and were shown to play a role in maintaining Hox expression once initiated (Takihara et al, 1997; Tomotsune et al, 2000). Therefore, the fact that rae28 expression in the heart is down-regulated during development and the expression of other PcG genes persists seems to indicate that molecular switching mechanisms work to change constituents of the PcG complexes. It is speculated that cardiomyocyte-specific overexpression of rae28 may eliminate formation of authentic PCG complexes later during development or that the persistent presence of early embryonic-type PcG complexes with rae28 may impair maintenance of cardiomyocytes that need to function throughout life. As previously reported, mutations in sarcomeric proteins that directly affect the contractile function are known to eventually result in hypertrophic cardiomyopathy, whereas mutations in cytoskeletal proteins that disrupt cell membrane stability and/or impair force transmission will be manifested as DCM. However, even mutations in a single cytoskeletal protein, cardiac $\alpha$-actin, result in either DCM or hypertrophic cardiomyopathy (Olson et al, 1998). Moreover, not only mutations in the sarcomeric and cytoskeletal proteins but also genetic modifications in signaling molecule expression can cause DCM or hypertrophic cardiomyopathy. Thus, a recent study of cardiomyopathy has revealed genetic and molecular complexity. Although it is still unclear which molecular pathways are regulated by PcG complexes to maintain cardiomyocytes, we have provided for the first time evidence suggesting that PCG constitute a novel transcription regulatory system for maintaining cardiomyocytes.

\section{Materials and Methods}

\section{Production of $\beta$ MHC-rae28 and RSV-rae28 Transgenic Mice}

Full-length rae28 cDNA, previously designated rae2819 (Nomura et al, 1994), was inserted downstream to a mouse $\beta M H C$ promoter at the Hpal site ( $\beta M H C-r a e 28)$ (Rindt et al, 1993) and was also inserted into the pmiwSV vector at the HindIII and Xbal sites (RSV-rae28) (Wakamatsu et al, 1997). A RSV enhancer and a chicken $\beta$-actin promoter were included in the pmiwSV vector to enhance transcription of the insert cDNA. Each of the plasmid DNAs was digested with BamHI together with Aatll or Notl to release the transgene DNA fragment, which was then separated from the vector by agarose gel electrophoresis. The 
DNA fragment was purified and microinjected by following standard procedures (Hogan et al, 1994). Founder animals of both lines were identified by PCR screening using primers for the rae28 cDNA (5'GGCAGAAGCAGATGGGAGTG-3' and 5'-GAGGGGCAGTGAGGGTTGTT-3'). Founder lines with the $B M H C$ rae28 transgene and those with the RSV-rae28 transgene were then genetically backcrossed to C57BL/6 strain mice to minimize the effect of genetic background before being used in this study. The numbers of transgene copies were determined by means of Southern blot analysis.

\section{Generation of rae28-Deficient Embryos Expressing Exogenous rae28}

Mice deficient in rae28 were generated and maintained by heterozygous intercrossing as described previously (Takihara et al, 1997). The transgenic mice were backcrossed to C57BL/6 strain mice as described above, and the resulting progeny were mated with heterozygous rae28-deficient mice $\left(\right.$ rae2 $\left.^{+/-}\right)$. After further backcrossing with rae $28^{+/-}$, embryonic progeny were obtained by cesarean section, and their genotypes were identified by means of PCR as described previously to obtain rae $28^{-1-}$ embryos carrying either of the transgenes. These embryos were then examined histologically and subjected to in situ hybridization analyses.

\section{Histologic Examination and TUNEL Assay}

Age-matched wild-type and transgenic mice were killed by cervical dislocation. The hearts were immediately removed from the mice and placed in $4 \%$ paraformaldehyde/phosphate buffered saline at $4^{\circ} \mathrm{C}$. They were infiltrated with paraffin and serially sectioned $(3$ to $5 \mu \mathrm{m}$ ) on a microtome (MicroM, Walldorf, Germany). Sections were stained with hematoxylin and eosin, and apoptosis was detected by using either the Annexin-V-Fluos staining kit (Roche, Mannheim, Germany) or the TUNEL assay. For the TUNEL assay, 3 '-end labeling of DNA with fluorescence-conjugated dUTP was performed with an in situ cell death detection kit (Boehringer Mannheim, Mannheim, Germany) following the manufacturer's instructions. For double staining experiments, the sections were stained simultaneously with 2,6-diamidino-2-phenylindole (Sigma, St. Louis, Missouri). The stained sections were examined under a fluorescent microscope (BX50; Olympus, Tokyo, Japan). Apoptosis was also confirmed by detecting internucleosomal cleavage of DNA. The homogenized myocardium was fixed in $70 \%$ ethanol and centrifuged, and the pellet was resuspended in a phosphate-citrate buffer. After centrifugation the supernatant was concentrated and subjected to gel electrophoresis (Liu et al, 1995).

\section{Whole-Mount In Situ Hybridization}

Whole-mount in situ hybridization analysis was performed as described previously (Tomotsune et al, 2000). Briefly, embryos at $9.5 \mathrm{dpc}$ were fixed in $4 \%$ paraformaldehyde/phosphate buffered saline and dehydrated through a methanol series in PBS containing $0.1 \%$ Tween-20. The samples were then rehydrated and bleached with $6 \%$ hydrogen peroxide. After treatment with $10 \mu \mathrm{g} / \mathrm{ml}$ proteinase K (Sigma), the samples were soaked in prewarmed prehybridization buffer (50\% formamide, $5 \times \mathrm{SSC}, 50 \mu \mathrm{g} / \mathrm{ml}$ yeast total RNA, $1 \%$ SDS, $50 \mu \mathrm{g} / \mathrm{ml}$ heparin) for 1 hour at $70^{\circ} \mathrm{C}$ and hybridized overnight with a digoxigenin-labeled riboprobe. The riboprobes were synthesized with digoxigenin-UTP, T3 or T7 polymerase (Boehringer Mannheim), and a rae28 cDNA as a template. The samples were washed in solution I ( $50 \%$ formamide, $5 \times$ SSC, $1 \%$ SDS) and in solution II (50\% formamide, $2 \times$ SSC) and in Tris-buffered saline (TBS) containing $0.1 \%$ Tween-20 (TBS-T). The samples were then treated with $10 \%$ heat-inactivated sheep serum in TBS-T containing $2 \mathrm{~mm}$ levamisole for 2 hours and incubated overnight in the preabsorbed antibody solution at $4^{\circ} \mathrm{C}$, followed by intensive washing. An alkaline phosphatase reaction was then performed in an NTMT solution [100 mM NaCl, $100 \mathrm{~mm}$ tris- $\mathrm{HCl}(\mathrm{pH}$ 9.5), $50 \mathrm{~mm} \mathrm{MgCl}_{2}, 0.1 \%$ Tween-20, $2 \mathrm{~mm}$ levamisol] containing $300 \mu \mathrm{g} / \mathrm{ml}$ 4-nitro blue tetrazolium chloride (Boehringer Mannheim) and $150 \mu \mathrm{g} / \mathrm{ml}$ 5-bromo-4chloro-3-indolylphosphate (Boehringer Mannheim). The embryos were dehydrated by means of a methanol series, and hybridization was observed under a microscope.

\section{Northern Blot Analysis and RT-PCR Assay}

Total cellular RNAs were prepared from the hearts by means of ISOGEN (Nippon Gene, Tokyo, Japan) and were subjected to Northern blot analysis as described previously (Nomura et al, 1994). For the RT-PCR assay, RNAs were treated with RNase-free DNase I (Boeringer Mannheim) and suspended in distilled water. The first-strand cDNA synthesis was performed with Super Script II reverse transcriptase (GIBCO$\mathrm{BRL}$, Gaithersburg, Maryland) and an oligo(dT 20$)$ primer according to the manufacturer's instructions. PCR amplification was performed with a PCR system (Perkin Elmer, Branchburg, New Jersey) using the previously described oligonucleotide primers specific for myosin light chain (MLC)-2v, $\alpha-M H C, \beta M H C, A N F$, and hypoxanthine-guanine phosphoribosyltransferase (HGPRT) (Conway et al, 1997; Kubalak et al, 1994; Lyons et al, 1995; Miller-Hance et al, 1993; Xu et al, 1998) and 5'-ACCGCTCTTGTGTGTGACAA-3', 5'-CAGGGCATAGCCCTCATAGA-3' for skeletal $\alpha$-actin, and 5'-TCTGGCGATGGTGTAACTCA-3', 5'-ATGGTGGTGCCTCCAGATAG-3' for cardiac $\alpha$-actin. We also used the following oligonucleotide primers to detect expression of PcG members: 5'-CAACAAAGGGGAAAAGCTGA-3' and 5'-ACATCCGTGACAAAGACGTG-3' for M33, 5'TTGGTCGAACTTAGCGTGTG- 3' and 5'-CCACTACAGGGTTTCCCAAA-3' for bmi1, 5'-AGTTCCTCCGCAACAAAATG- $3^{\prime}$ and 5'-GGAGCGCCATTAACAGTCAT- $3^{\prime}$ for mel18, the same oligonucleotide primers used for genotyping as described above for rae28, 5'-CACTGGCATCTCCAGGTTTT-3' and 5'- 
GAGGTATGGGGAAGGGGTTA-3' for mph2, 5'-AGCCACCATCACCTTCCCACAACTT- 3 ' and 5'-ACTGCCAGGCTTGGGACCTCTCTTC-3' for scmh1, and 5'ATGTTCCAGTATGACTCCACTCACG-3' and 5'GAAGACACCAGTAGACTCCACGACA-3' for glyceraldehyde-3-phosphate dehydrogenase (GAPDH). The PCR cycles consisted of the following: $95^{\circ} \mathrm{C}$ for 5 minutes, followed by 25 to 40 cycles at $95^{\circ} \mathrm{C}$ for 1 minute, $55^{\circ} \mathrm{C}$ for 1 minute, and $72^{\circ} \mathrm{C}$ for 1 minute. The PCR products were electrophoresed on $1 \%$ agarose gels and stained with ethidium bromide $(0.5 \mu \mathrm{g} / \mathrm{ml})$. The products were also blotted to nylon membranes after electrophoresis and hybridized with radiolabeled genespecific probes to confirm the specificity of the products.

\section{Echocardiography}

Mice were anesthetized by intraperitoneal injection of a mixture of ketamine $(100 \mathrm{mg} / \mathrm{kg})$ and xylazine $(5$ $\mathrm{mg} / \mathrm{kg}$ ). Echocardiography used a SSA-390A ultrasound system equipped with a $15-\mathrm{MHz}$ transducer (Toshiba, Nasu, Japan). A two-dimensionally guided $M$-mode echocardiogram of the left ventricle was recorded on S-VHS video (SVO-9500MD; Sony, Kosei, Japan). LV end-diastolic and end-systolic dimensions and interventricular septal thickness, LV posterior wall thickness, and ejection fraction were measured. Heart rates were monitored by electrocardiogram. The quantitative measurements represented consensus estimated by two different investigators (H.K. and Y.K.) with an interobserver variability of $<5 \%$. Statistic analyses were performed using unpaired Student's $t$ tests, and resultant values were considered significant at $p<0.05$.

\section{Electron Microscopy}

Heart samples were fixed in a mixture of $3 \%$ paraformaldehyde and $3 \%$ glutaraldehyde in a $0.1 \mathrm{~m}$ cacodylate buffer and postfixed in $1 \%$ osmium tetroxide. The samples were then stained en bloc in uranylacetate, dehydrated through an ethanol series, and embedded in Epon 812. Semi-thin sections with a thickness of 0.5 $\mu \mathrm{m}$ were stained with toluidine blue for light microscopic examination. After careful identification of location and orientation of the samples, ultrathin sections were prepared, doubly stained with uranylacetate and lead citrate, and observed under a JEOL 2000EX electron microscope (Kumai et al, 2000).

\section{Western Blot Analysis}

Tissues were lysed and the clarified extracts were prepared as described previously (Takihara et al, 1997). Equivalent amounts of total protein were separated by SDS-PAGE on a $10 \%$ gel and transferred to an Immobilon membrane (Millipore, Bedford, Massachusetts). The membrane was stained with a polyclonal antibody against rae28 as described previously (Takihara et al, 1997).

\section{Acknowledgements}

We thank Drs. J. Robbins and $\mathrm{H}$. Kondoh for the gifts of a $\beta M H C$ promoter and a pmiwSV vector, respectively, and Ms. R. Hasegawa for secretarial help. We are grateful for the extensive use of the Genome Information Research Center and Department of Science for Laboratory Animal Experimentation, Osaka University.

\section{References}

Akasaka T, Kanno M, Balling R, Mieza MA, Taniguchi M, and Koseki H (1996). A role for mel-18, a Polycomb group related vertebrate gene, during the anteroposterior specification of the axial skeleton. Development 122:1513-1522.

Alkema MJ, Brock M, Verhoeven E, Otte A, Veer LJvt, Berns A, and van Lohuizen M (1997). Identification of Bmi1interacting proteins as constituents of a multimeric mammalian Polycomb complex. Genes Dev 11:226-240.

Conway SJ, Henderson DJ, and Copp AJ (1997). Pax3 is required for cardiac neural crest migration in the mouse: Evidence from the splotch $(\mathrm{Sp} 2 \mathrm{H})$ mutant. Development 124:505-514.

Córe N, Bel S, Gaunt SJ, Aurrand-Lions M, Pearce J, Fisher $A$, and Djabali M (1997). Altered cellular proliferation and mesoderm patterning in Polycomb-M33-deficient mice. Development 124:721-729.

del Mar Lorente M, Marcos-Gutierrez C, Perez C, Schoorlemmer J, Ramirez A, Magin T, and Vidal M (2000). Loss- and gain-of-function mutations show a Polycomb group function for Ring1A in mice. Development 127:5093-5100.

Ghadially FN (1988). Ultrastructural pathology of the cell and matrix. London: Butterworths, 266-277.

Hashimoto N, Brock HW, Nomura M, Kyba M, Hodgson J, Fujita Y, Takihara $Y$, Shimada $K$, and Higashinakagawa T (1998). RAE28, BMI1, and M33 are members of heterogeneous multimeric mammalian Polycomb group complexes. Biochem Biophys Res Commun 245:356-365.

Hogan B, Beddington R, Costantini F, and Lacy E (1994). Manipulating the mouse embryo; A laboratory manual, 2nd ed. Cold Spring Harbor, New York: Cold Spring Harbor Laboratory Press.

Katoh-Fukui Y, Tsuchiya R, Shiroishi T, Nakahara Y, Hashimoto N, Noguchi K, and Higashinakagawa T (1998). Maleto-female sex reversal in M33 mutant mice. Nature 393:688692.

Kubalak SW, Miller-Hance WC, O'Brien TX, Dyson E, and Chien KR (1994). Chamber specification of atrial myosin light chain-2 expression precedes septation during murine cardiogenesis. J Biol Chem 269:16961-16970.

Kumai M, Nishii K, Nakamura K, Takeda N, Suzuki M, and Shibata $Y$ (2000). Loss of connexin45 causes a cushion defect in early cardiogenesis. Development 127:3501-3512.

Liu Y, Cigola E, Cheng W, Kajstura J, Olivetti G, Hintze TH, and Anversa P (1995). Myocyte nuclear mitotic division and programmed myocyte cell death characterize the cardiac myopathy induced by rapid ventricular pacing in dogs. Lab Invest 73:771-787.

Lyons I, Parsons LM, Hartley L, Li R, Andrews JE, Robb L, and Harvey RP (1995). Myogenic and morphogenetic defects 
in the heart tubes of murine embryos lacking the homeo box gene Nkx2-5. Genes Dev 9:1654-1666.

Miller-Hance WC, LaCorbiere M, Fuller SJ, Evans SM, Lyons G, Schmidt C, Robbins J, and Chien KR (1993). In vitro chamber specification during embryonic stem cell cardiogenesis: Expression of the ventricular myosin light chain-2 gene is independent of heart tube formation. J Biol Chem 268:25244-25252.

Nomura M, Takihara Y, and Shimada K (1994). Isolation and characterization of retinoic acid-inducible cDNA clones in F9 cells: One of the early inducible clones encodes a novel protein sharing several highly homologous regions with a Drosophila polyhomeotic protein. Differentiation 57:39-50.

Olson TM, Michels VV, Thibodeau SN, Tai YS, and Keating MT (1998). Actin mutations in dilated cardiomyopathy, a heritable form of heart failure. Science 280:750-752.

Osugi T, Shirai M, Koga H, Nishiguchi S, Yamauchi-Takihara K, Takihara Y (2000). rae28, a member of the mammalian Polycomb group genes, is required for maintenance of Nkx2.5 expression in cardiac development. Circulation 102(Suppl):II-99.

Rindt H, Gulick J, Knotts S, Neumann J, and Robbins J (1993). In vivo analysis of the murine beta-myosin heavy chain gene promoter. J Biol Chem 268:5332-5338.

Schumacher A, Faust C, and Magnuson T (1996). Positional cloning of a global regulator of anterior-posterior patterning in mice. Nature 383:250-253.

Shao Z, Raible F, Mollaaghababa R, Guyon JR, Wu C, Bender W, and Kingston RE (1999). Stabilization of chromatin structure by PRC1, a Polycomb complex. Cell 98:37-46.

Srivastava D and Olson EN (2000). A genetic blueprint for cardiac development. Nature 407:221-226.

Takihara $\mathrm{Y}$ and Hara J (2000). The Polycomb-group genes and hematopoiesis. Int $\mathrm{J}$ Hematol 72:165-172.
Takihara Y, Tomotsune D, Shirai M, Katoh-Fukui Y, Nishii K, Motaleb MA, Nomura M, Tsuchiya R, Fujita Y, Shibata Y, Higashinakagawa T, and Shimada K (1997). Targeted disruption of the mouse homologue of the Drosophila polyhomeotic gene leads to altered anteroposterior patterning and neural crest defects. Development 124:3673-3682.

Tashiro A, Masuda T, and Segawa I (1990). Morphometric comparison of mitochondria and myofibrils of cardiomyocytes between hypertrophic and dilated cardiomyopathies. Virchows Arch A Pathol Anat Histopathol 416:473-478.

Tomotsune D, Shirai M, Takihara Y, and Shimada K (2000). Regulation of Hoxb3 expression in the hindbrain and pharyngeal arches by rae28, a member of the mammalian Polycomb group of genes. Mech Dev 98:165-169.

Tomotsune D, Takihara Y, Berger J, Duhl D, Joo S, Kyba M, Shirai M, Ohta H, Matsuda Y, Honda BM, Simon J, Shimada K, Brock HW, and Randazzo F (1999). A novel member of murine Polycomb-group proteins, Sex comb on midleg homolog protein, is highly conserved, and interacts with RAE28/mph1 in vitro. Differentiation 65:229-239.

van der Lugt MT, Domen J, Linders K, Roon M, RobanusMaandag E, Riele H, Valk M, Deschamps J, Sofroniew M, van Lohuizen M, and Berns A (1994). Posterior transformation, neurological abnormalities, and severe hematopoietic defects in mice with a targeted deletion of the bmi- 1 protooncogene. Genes Dev 8:757-769.

Wakamatsu $\mathrm{Y}$, Watanabe $\mathrm{Y}, \mathrm{Nakamura} \mathrm{H}$, and Kondoh $\mathrm{H}$ (1997). Regulation of the neural crest cell fate by $\mathrm{N}$-myc: Promotion of ventral migration and neuronal differentiation. Development 124:1953-1962.

Xu C, Liguori G, Adamson ED, and Persico MG (1998). Specific arrest of cardiogenesis in cultured embryonic stem cells lacking Cripto-1. Dev Biol 196:237-247.

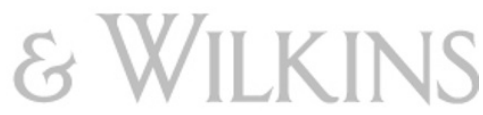

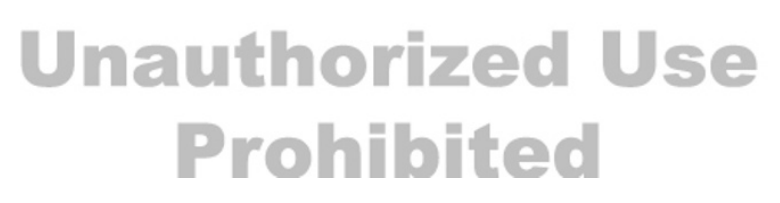

Laboratory Investigation $\bullet$ April $2002 \bullet$ Volume $82 \bullet$ Number 4 